\title{
High Order Sliding Mode Control for the Aircraft Antiskid Braking System
}

\author{
Longfei FU, Guanglai TIAN, Yuren LI, Bo LIANG, Hongling WANG \\ School of Automation, Northwestern Polytechnical University, Xi'an 710072, China
}

\begin{abstract}
A high order sliding mode control (HOSMC) algorithm is proposed to control the nonlinear system with parameter uncertainty in this paper, with the purpose of the chattering avoidance. According to the aircraft antiskid braking system(ABS) model, a HOSMC algorithm is presented and a bipolar sigmoid function is developed. The stability and convergence of proposed algorithm is analyzed with Lyapunov theory in finite time. The results indicate that the HOSMC algorithm has a better performance and chattering avoidance than the conditional ones. Additionally the maximum friction coefficient is acquired in a short time, and the optimal slip ratio is achieved under the runway simulation.
\end{abstract}

KEYWORD: high order sliding mode; slip ratio; anti-skid braking system; aircraft

\section{INTRODUCTION}

The aircraft ABS can control the braking pressures according to the adhesion between the wheel and track, indicated by friction coefficient. Thus the friction coefficient is a key factor, good controlling of $\mathrm{ABS}$ is crucial in maintaining the maximum friction coefficient, increase the friction coefficient, and decrease the braking distance. The braking performance and safety can also be improved[1].

Sliding mode control has a strong robustness for its insensitive to parameters and resistance to disturbance[2]. However, due to the existence chattering phenomenon the application of sliding mode control face challenge. This year, high order sliding mode control has become the focus in the fields, it not only keeps the traditional advantages of strong robustness, but also eliminates chattering phenomenon which is the core problem of traditional[3-5].

The high order sliding mode is a essence continuous, it can eliminate system chattering without the expense of the robustness of the controller. References [2-4] proposed a method of high order sliding mode controller using the integral sliding mode for uncertain nonlinear systems ensuring robust tracking performance and finite time theory is used. Reference [5] developed a higherorder sliding mode observer based on super twisting algorithm for state and unknown input estimations. Reference [6] proposed SMC using discontinuous sign function act on the time derivative of the control input. Reference [7] presented a second order sliding mode traction controller for road vehicles during skid braking and spin acceleration. Reference [8] proposed HOSMC for a highly complex nonlinear system suffering from the mismatched type of uncertainty caused by varying aerodynamic parameters and environmental disturbance in vertical take-off and landing aircraft system. References [910] designed a first-order sliding mode observers in the friction state. Reference [11] gave the nonlinear SMC to estimate the optimal slip ratio. Reference [12] designed a hybrid controller based on a sliding mode controller and fuzzy controller, proposed three different sliding mode controllers and fuzzy controller is employed to eliminate the oscillation.

In this paper, a controller is systematic designed based on the HOSMC for nonlinear systems with parameter uncertainties and external disturbances. The algorithm based on slip ratio which is derived from the fuselage motion to improve the robustness of SMC algorithm and chattering phenomenon. The HOSMC asymptotical stability is achieved and the algorithm is verified with the aircraft ABS model on the dry runway condition.

\section{PROBLEM FORMULATION}

According to Newton's law and moment balance principle, the aircraft fuselage ground dynamics model is shows as equation (1), for the simplify like[1],[11]: 
a. The fuselage and landing gear are regarded as a rigid thing;

b. The earth is regarded as the inertial coordinate system.

$$
\begin{aligned}
\dot{x}_{1}= & \frac{T_{0}+k_{v} x_{1}}{M}-\frac{\rho C_{d} S x_{1}^{2}}{2 M}-\frac{\rho C_{p} S_{p} x_{1}^{2}}{2 M} \\
& -\frac{\mu\left[\left(a-u_{2} h\right)\left(g-\frac{\rho C_{L} S x_{1}^{2}}{2 M}\right)+h_{t} \frac{T_{0}+k_{v} x_{1}}{M}+h_{p} \frac{\rho C_{p} S_{p} x_{1}^{2}}{2 M}\right]}{a+b+\left(u-u_{f}\right) h} \\
& -\frac{u_{2}\left[(b+u h)\left(g-\frac{\rho C_{L} S x_{1}^{2}}{2 M}\right)-h_{t} \frac{T_{0}+k_{v} x_{1}}{M}-h_{p} \frac{\rho C_{p} S_{p} x_{1}^{2}}{2 M}\right]}{a+b+\left(u-u_{2}\right) h}
\end{aligned}
$$

Where $f_{1}=\mu N_{1}$ and $f_{2}=\mu_{2} N_{2}, \mu, \mu_{2}$ represent main wheel and front wheel friction coefficient respectively, $V_{X}, V_{Y}, M, \theta$ represent longitudinal velocity, vertical velocity, mass and pitch angle respectively. The other parameters in formula (1) are $k_{v}, h, h_{s}, h_{t}$ which represent engine thrust coefficient, the height of fuselage center, the distance of engine thrust from horizontal axis and the distance of drag parachute from horizontal axis respectively. The state parameter $x_{1}$ is the longitudinal velocity $V_{X}$.

The dynamics model that describe the wheel behavior is obtained as follows

$$
J \dot{\omega}=T_{j}-T_{b}
$$

The dynamic wheel radius can describe as

$R_{v b}=R-\frac{1}{4} k_{\sigma} N_{1}$

Where $T_{j}=R_{v b} f$ represent friction torque and $T_{b}=k_{b} P_{b}$ represent braking torque, $\omega, J, P_{b}, R_{v b}$, $R, \quad K_{\sigma}, f$ represent angular velocity, rotating inertia, brake force, dynamic radius, static radius, compression coefficient of wheel and friction force of the wheel respectively.

Let $x_{2}=\omega$, then

$$
\begin{aligned}
\dot{x}_{2}= & \frac{R_{v b} \mu\left[\left(a-u_{2} h\right)\left(m g-\frac{\rho C_{L} S x_{1}^{2}}{2}\right)+h_{t}\left(T_{0}+k_{v} x_{1}\right)+h_{p} \frac{\rho C_{p} S_{p} x_{1}^{2}}{2}\right]}{\left.n I\left(a+b+\left(u-u_{2}\right) h\right)\right)} \\
& +\frac{k_{b}}{I} P_{b}
\end{aligned}
$$

The friction coefficient can describe as

$$
\mu=D \sin (C a \tan (B \sigma))
$$

The slip ratio can describe as

$$
\sigma=\frac{V_{X}-R \omega}{V_{X}}=\frac{x_{1}-R x_{2}}{x_{1}}
$$

\section{HIGH ORDER SLIDING MODE CONTROL}

In general, we consider the following nonlinear system:

$$
\left\{\begin{array}{l}
\dot{\mathbf{x}}=f(\mathbf{x})+g(\mathbf{x}) u \\
y=h(\mathbf{x})
\end{array}\right.
$$

Where $\mathbf{x} \in \mathfrak{R}^{n}$ is a vector of measurable states, $u \in \mathfrak{R}$ is the input control, $f(\mathbf{x})$ and $g(\mathbf{x})$ are known smooth nonlinear functions and $\mathrm{g}(\mathrm{x})$ is full rank for all $\mathbf{x} \in \mathfrak{R}^{n}, h(\mathbf{x})$ is the output function.

Assume, the system relative degree is 1 . The high order sliding mode control law proposes the super twisting algorithm[5].

$$
u=-\lambda|x|^{\frac{1}{2}} \operatorname{sgn}(x)-\alpha \operatorname{sgn}(x)
$$

Where $\lambda>0$ and $\alpha>0$ are some chosen constants, $\operatorname{sgn}(\bullet)$ is the symbolic function. The principle of super twisting algorithm is shown as figure 1.

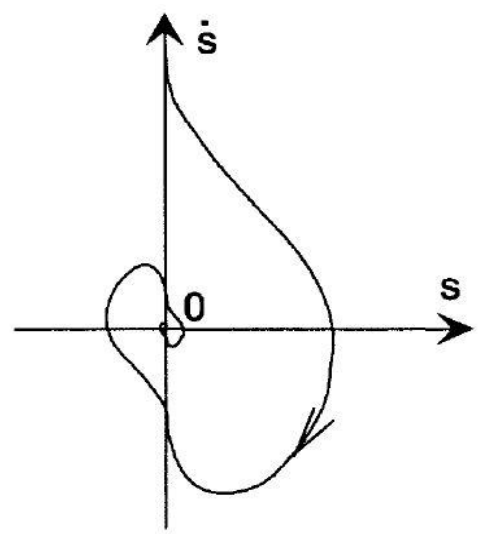

Figure. 1 The principle of super twisting algorithm`

Definition the sliding mode surface is $s=h(\mathbf{x})$. Choosing a Hurwitz function $A$, for any positive definite symmetric matrix $Q$, there must exist a positive definite symmetric matrix $P$ meet the Lyapunov equation.

$$
A^{T} P+P A=-Q
$$

Taking the Lyapunov function

$$
V=\zeta^{T} P \zeta
$$

Where $\zeta^{T}=\left[\lambda|x|^{\frac{1}{2}} \operatorname{sgn}(x) \quad \alpha \operatorname{sgn}(x)\right], \quad V \quad$ is the continuous positive definite function and derivable. Derivate the $V$, we can obtain follows.

$$
\begin{gathered}
\dot{V}=\frac{1}{|\zeta|} \zeta^{T}\left(A^{T} P+P A\right) \zeta=-\frac{1}{|\zeta|} \zeta^{T} Q \zeta \\
\eta_{\min }(P)\|\zeta\|_{2}^{2} \leq \zeta^{T} P \zeta \leq \eta_{\max }(P)\|\zeta\|_{2}^{2}
\end{gathered}
$$


Where $\eta_{\min }$ and $\eta_{\max }$ are the minimum and maximum eigenvalue of the matrix $P$.

From (11)-(12) has

$$
\begin{aligned}
\dot{V} & \leq-\frac{1}{|\zeta|} \eta_{\min }(Q)\|\zeta\|_{2}^{2} \\
& =-\frac{\|\zeta\|_{2}}{|\zeta|} \eta_{\min }(Q)\|\zeta\|_{2} \\
& \leq-\eta_{\min }(Q)\|\zeta\|_{2} \\
& \frac{V^{\frac{1}{2}}}{\eta_{\max }^{\frac{1}{2}}(P)} \leq\|\zeta\|_{2}
\end{aligned}
$$

Then, the equation meet

$$
\dot{V} \leq-\frac{\eta_{\min }(Q)}{\eta_{\max }^{\frac{1}{2}}(P)} V^{\frac{1}{2}}
$$

$\dot{V}$ is negative semidefinite and the convergent condition is satisfied.

\section{SIMULATION}

The proposed HOSMC is applied to the nonlinear system of aircraft ABS and the objective is to control the slip ratio optimal. The parameters used in the simulation are given in Table 1 .

Table 1. The aircraft parameters

\begin{tabular}{|c|c|}
\hline Parameter & Value(Unit) \\
\hline Aircraft initial velocity $V_{X}$ & $72(\mathrm{~m} / \mathrm{s})$ \\
\hline Aircraft mass M & $17000(\mathrm{~kg})$ \\
\hline Tyre radius R & $0.4(\mathrm{~m})$ \\
\hline Rotational inertia of the tyre I & $12\left(\mathrm{~kg} \cdot \mathrm{m}^{2}\right)$ \\
\hline Braking coefficient $k_{b}$ & 1.2 \\
\hline
\end{tabular}

In order to indicate the performance of we devised control policy, the high order sliding mode controller is applied to ABS of aircraft for numerical simulations. On the side, the performance of the traditional sliding mode controller is also provided for the purpose of comparison. Two control strategy on the aircraft ABS will show some significant results.

The results of the variations of aircraft velocity and wheel velocity are shown in Figure 2. The aircraft velocity and wheel velocity reduce gradually according to slip ratio with time, respectively. As seen for Figure 2, the braking time with HOSMC strategy is $12.6 \mathrm{~s}$ which is shorter than SMC strategy that is $14.4 \mathrm{~s}$. So, according to the braking time the HOSMC achieves a better effect.

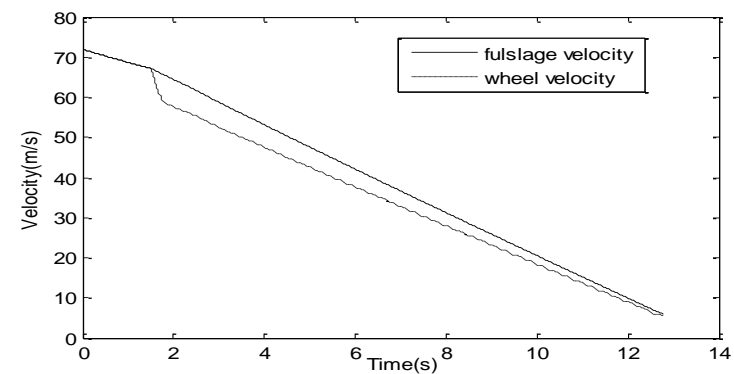

(a) HOSMC algorithm

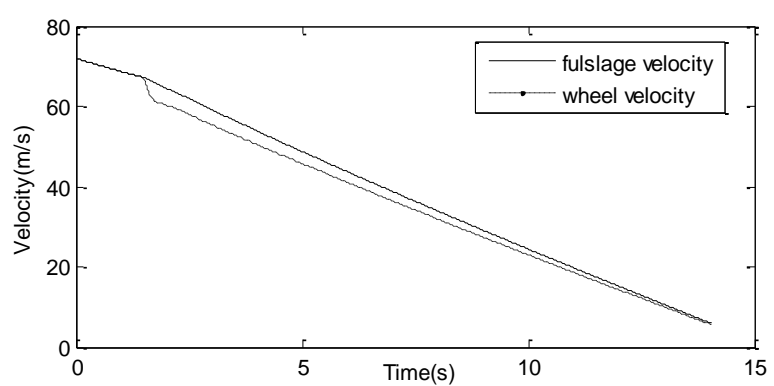

(b) SMC algorithm

Figure 2. The variations of velocities

The results of the friction coefficient are shown in Figure 3. As seen for Figure 3, the friction coefficient with HOSMC strategy reaches the actual value 0.8 quickly and keeps at it and the SMC strategy is about 0.722 , a little smaller than the former and decreasing with time. The lower friction coefficient result in a long braking time.

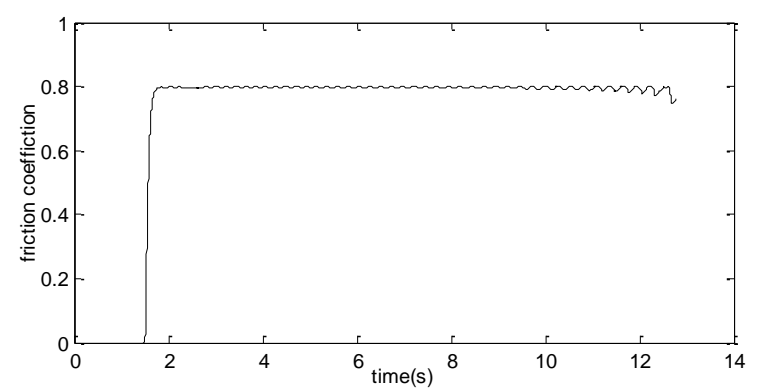

(a)HOSMC algorithm

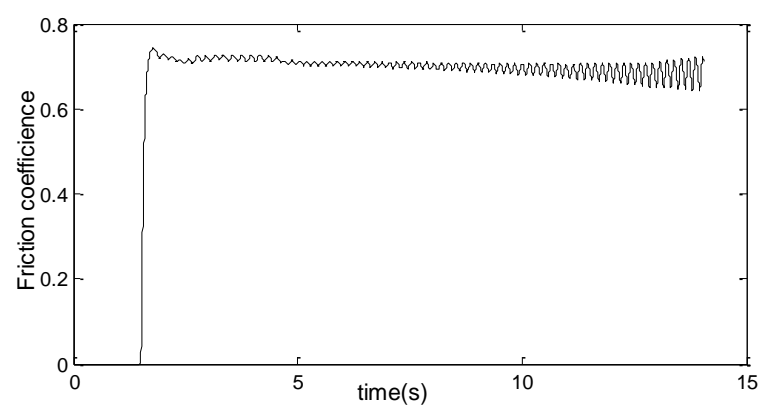

(b) SMC algorithm

Figure 3. Friction coefficient

It can be seen from Figure 4 that the slip ratio using proposed HOSMC can reaches the optimum value 0.115 with shortest time, compared to the SMC which is 0.065 . The reason of magnitude of slip ratio is larger at the low velocity, is that the slip 
ratio approach infinity when aircraft velocity and wheel velocity approach zero.

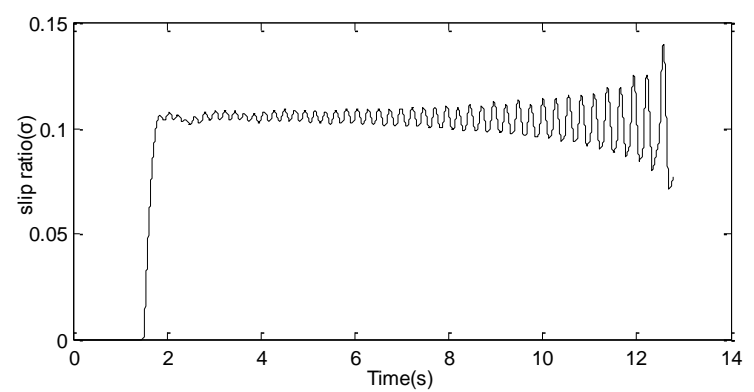

(a) HOSMC algorithm

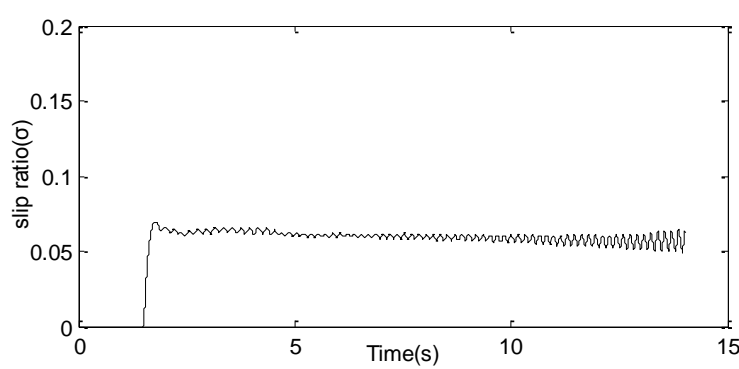

(b) SMC algorithm

Figure 4. Slip ratio

\section{CONCLUSIONS}

In this work, a high order sliding mode controller was proposed for uncertainty and nonlinear with application to aircraft ABS. The aircraft fuselage ground dynamics model and wheel behavior model are derived. A HOSMC algorithm is presented to control the slip ratio to the desired range. The convergence of the designed sliding mode is proven.

The proposed aircraft ABS controller has a strong robustness and chattering avoidance, furthermore it is more effective than traditional ones and simulation results highlights the validation and advantages.

\section{REFERENCES}

[1] Fu Longfei, Tian Guanglai,et al. Designing an effective sliding mode variable structure control law with feedback linearization of aircraft anti-skid braking system. Journal of Northwestern Polytechnical University, 2013, 31(6): 962-966.

[2] Salah Laghrouchea, Franck Plestanb, Alain Glumineaub. Higher order sliding mode control based on integral sliding mode. Automatica. 2007, 43:531 - 537.

[3] Rios, H., Davila, J., Fridman, L. High-order sliding mode observers for nonlinear autonomous switched systems with unknown inputs, J. Franklin Inst., 2012, 349(10): 2975-3002.

[4] Moreno, J.A. A Lyapunov approach to second-order sliding mode controllers and observers. Proc. of 47 th IEEE Conf. on Decision and Control. 2008,12: 28562861.

[5] Jagat Jyoti Rath, Kalyana Chakravarthy Veluvolu, Michael Defoort. Higher order sliding mode observer for estimationof tyre friction in ground vehicles. IET Control Theory Appl., 2014, 8(6): 399-408.

[6] Sanjoy MONDAL, Chitralekha MAHANTA. Adaptive integral higher order sliding mode controller for uncertain systems. J Control Theory Appl, 2013, 11 (1): 61-68.

[7] Matteo Amodeo, Antonella Ferrara. Wheel Slip Control via Second Order Sliding Modes Generation. 46th IEEE Conference on Decision and Control. 2007,12:3889-3894.

[8] Sanjoy Mondal, Gokul T.V, Chitralekha Mahanta. Adaptive Second Order Sliding Mode Controller for Vertical Take-off and Landing Aircraft System. IEEE 7th International Conference on Industrial and Information Systems. 2012.

[9] Xie, W.F. Sliding mode observer based adaptive control for servo actuator with friction. IEEE Trans. Ind. Electron., 2007, 54 (3): 1517-1527.

[10] Lee, D., Park, Y. Sliding-mode-based parameter identification with application to tire pressure and tireroad friction, Int. J. Autom. Technol., 2011, 12 (4):571577.

[11] Fu Longfei, Li Yuren, et al. Slid mode VSC for aircraft anti-skid braking system with index reaching law. Applied Mechanics and Materials, 2013, v 336-338: 973-977.

[12] Habibi, Mehran, Yazdizadeh, Alireza. A Novel FuzzySliding Mode Controller for Antilock Braking System. Proceedings 2nd IEEE International Conference on Advanced Computer Control, ICACC 2010, 4: 110-114. 